\title{
High-Energy Sky Observation by Two Small Satellites Using Formation Flight (FFAST)
}

\author{
Naohisa Anabuki ${ }^{1}$, Hiroshi Tsunemi ${ }^{1}$, Hiroshi Nakajima ${ }^{1}$, Kiyoshi Hayashida ${ }^{1}$, Hideyo Kunieda ${ }^{2}$, \\ Masayuki Itoh $^{3}$, Masanobu Ozaki ${ }^{4}$, Isao Kawano ${ }^{5}$, Toru Yamamoto ${ }^{5}$, Shinji Mitani ${ }^{5}$, \\ and FFAST team \\ ${ }^{1}$ Department of Earth and Space Science, Osaka University, Osaka, Japan \\ ${ }^{2}$ Department of Astrophysics, Nagoya University. Nagoya, Japan \\ ${ }^{3}$ Graduate School of Human Development and Environment, Kobe University, Kobe, Japan \\ ${ }^{4}$ Institute of Space and Astronautical Science, JAXA, Kanagawa, Japan \\ ${ }^{5}$ Institute of Aerospace Technology, JAXA, Tsukuba, Japan \\ E-mail(NA): anabuki@ess.sci.osaka-u.ac.jp
}

\section{Abstract}

We are planning to have a Formation Flying Astronomical Survey Telescope (FFAST) that will make a hard X-ray $(\leq 80 \mathrm{keV})$ imaging survey. Two small satellites $\left(\sim 1 \mathrm{~m}^{3}\right)$, a telescope satellite and a detector satellite, will be placed into a circular $550 \mathrm{~km}$ orbit and in formation flying that will be controlled the separation of $20 \mathrm{~m} \pm 10 \mathrm{~cm}$. With a relative-circular orbit, we can select the scanning sky region arbitrarily; in the case of covering $\Omega \sim 1000 \mathrm{deg}^{2}$ areas of sky, the detection limit of $0.1 \mathrm{mCrab}$ will be achieved in two years. Hence, the FFAST will be able to fill the sensitivity gap of AGN surveys performed by ongoing and future hard X-ray missions.

KEY WORDS: workshop: proceedings — Hard X-ray — Formation Flying — AGN survey

\section{Introduction}

The recent technological advances of multilayer coating enable us to develop focusing hard X-ray system using Brag reflection. In the last ten years, several research groups have conducted hard X-ray observations by a balloon-borne experiment, InFOCus, HERO, HEFT, and SUMIT. And, X-ray astronomical satellites that carry a hard X-ray telescope are being planned in the 2010 s (e.g. NuSTAR, ASTRO-H ). Due to the grazing angle of the X-ray reflection, on the other hand, the $\mathrm{X}$-ray telescope increase in length so that we can have enough effective area. However, the longer focal length will have a difficulty to be equipped with a single satellite. Hence, it is quite reasonable to separate the mirror and the detector into two spacecrafts maintained a certain distance using formation flying.

Therefore, we propose a Formation Flying Astronomical Survey Telescope (FFAST) mission that will cover a large sky area in hard X-ray band.

\section{Design and Performance}

The telescope satellite and the detector satellite of FFAST are put into the circular $550 \mathrm{~km}$ orbit and kept the distance of $20 \mathrm{~m} \pm 10 \mathrm{~cm}$ by a formation-flying guidance system. Fig.1 shows the configuration of FFAST in the orbit. The size of each satellite except the solar panels is about $1 \mathrm{~m}^{3}$.

The telescope satellite has a hard X-ray super-mirror that is an assembly of depth-graded $\mathrm{Pt} / \mathrm{C}$ multilayer coated mirrors in a conical approximation to the Wolter I geometry (Ogasaka et al. 2008). The FFAST X-ray telescope (FFAST/XRT) that covers the energy range up to $80 \mathrm{keV}$ is identical that of $A S T R O-H$ with the exception of the focal length. We have improved multiple thin-foil optics and now achieved $90 \operatorname{arcsec}(\mathrm{HPD})$ with good rotation asymmetry PSF. We show the effective area of FFAST/XRT in the middle left panel of Fig.1.

The detector satellite carries an SD-CCD that functions as a hard X-ray imaging spectrometer up to $100 \mathrm{keV}$ (Miyata and Tamura 2003). The SD-CCD is a CCD with a scintillator plate that is directly attached to the back-side of the CCD. X-rays enter into the CCD from the front (electrode) side. High energy X-rays that penetrate the CCD will be absorbed in the scintillator and converted into visible light, which is detected by the CCD. Note that the scintillator events, X-ray events directly detected in the depletion layer, and a cosmic-ray events are easily distinguished by the charge spread and the pulse height. The CCD chip is fully depleted which can be a back-illuminated CCD identical to that em- 

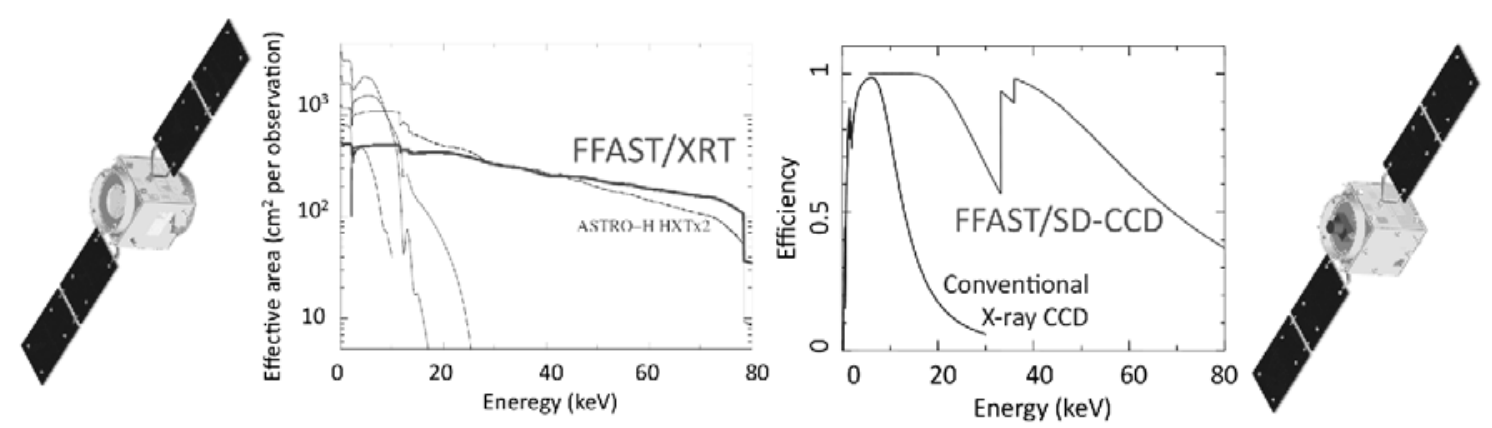

Fig. 1. FFAST configuration in orbit. (left : the telescope satellite, right : the detector satellite). The effective area of the FFAST/XRT (middle left). The detection efficiency of the SD-CCD (middle right).

ployed on ASTRO-H. The middle right panel of Fig.1 shows the detection efficiciency of the SD-CCD; the depletion depth of the CCD is $\sim 200 \mu \mathrm{m}$, and the thickness of the CsI scintillator plate is $\sim 300 \mu \mathrm{m}$. Three 3 -sidebuttable SD-CCD chips on the FFAST focal plane cover the FOV of $10^{\prime} \times 15^{\prime}$. And they are working at $-90^{\circ} \mathrm{C}$ with a mechanical cooler that is almost identical to that developed for the $A S T R O-H \mathrm{CCD}$ and that employed in the Suzaku satellite (Mitsuda et al. 2007).

We have already demonstrated the hard X-ray telescope with the super-mirror and the SD-CCD by SUMIT balloon-borne experiment in 2006 .

In the attitude control, the telescope satellite aligns the optical axis on the center of SD-CCD array, and the the detector satellite tracks the telescope to focus on the SD-CCD surface. Moreover, the roll angle of the detector satellite is controlled so that star images move in the same as vertical charge-transfer direction of the CCD, which is operated in TDI mode. We note that the detector satellite has a reaction control system (RCS) that can control the satellite orbit. The RCS is used to compensate the non-gravity perturbation except for contingency. Because the two satellites in a tandem construction fit inside shroud of a solid rocket now being developed, they will be launched by means of a single rocket.

\section{FFAST Orbital Design}

As has been mentioned, two satellites will be placed into Keplerian orbit and maintained a constant distance. According to the C-W solution of Hill's equation that describes the relative motion of these two spacecrafts, one can derive only two possible orbits for the formation flying, an along-track orbit and a relative-circular-orbit. Using a guiding-formation-flying with a small amount of thruster gas in the relative-circular orbit (relativecircular FF), we can arbitrarily choose and confine sky survey region. Thus, from the perspective of scientific objectives, we are considering employing the relativecicular FF.

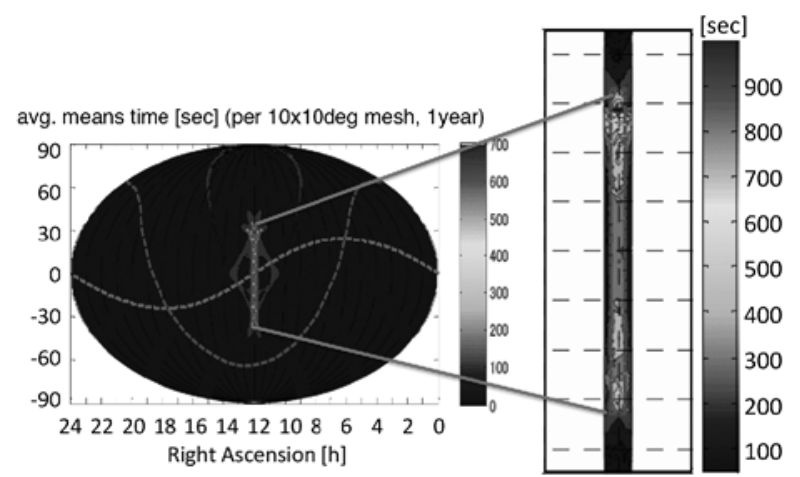

Fig. 2. Exposure map of FFAST $3 \times 66$ square degree scanning observation in a year.

And, we have designed the orbit for $3 \times 66$ square degree scanning observation. The expected exposure map in a year is shown in Fig.2. From our calculation, the survey flux limit of $0.1 \mathrm{mCrab}$ intensity will be achieved for $46 \%$ of the region. In two years, $0.1 \mathrm{mCrab}$ flux limit over $\sim 1000$ square degree of the sky. Hence, FFAST has a possibility to perform a unique AGN survey to fill the sensitivity gap of AGN surveys done by on-going and future missions in hard X-ray regime.

\section{Summary}

We described here the FFAST project that will be the first focusing hard X-ray mission using a formation flying. The FFAST survey in two years $\left(\Omega \sim 1000 \mathrm{deg}^{2}, S_{\text {lim }}\right.$ $\sim 0.1 \mathrm{mCrab}$ ) is expected to fill a sensitivity gap between shallow all-sky and deep pencil-beem AGN surveys.

\section{References}

Mitsuda et al. 2007, PASJ, 59S, 1

Miyata E. and Tamura K. 2003, JJAP, 42 L1201

Ogasaka et al. 2008, Proc. of SPEI, 7011, 19

Tsunemi H. et al. 2005, Proc. of SPIE, 5900, 184

Tsunemi H. 2005, NIMA, 541, 295 